\section{The Launch of the \\ International Journal of \\ Digital Health: Ensuring \\ Digital Transformation in \\ Healthcare Beyond Covid-19}

KEVIN YI-LWERN YAP (D)

JILAN LIU

THOMAS FRANCHI (D)

RIAZ AHMED AGHA (D)

*Author affiliations can be found in the back matter of this article

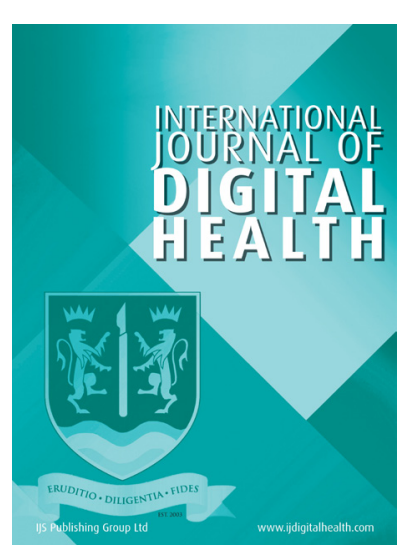

EDITORIAL

\title{
ABSTRACT
}

In recent years, digital health technologies have been evolving rapidly and there has been much interest and excitement surrounding their use in healthcare. The Covid-19 pandemic has brought about a significant jump in the adoption of digital health innovations worldwide, and healthcare providers are now experiencing a tremendous increase in the number of patients seen through telehealth. Further, many organizations are rethinking their strategies to find better ways to adapt and improve the health ecosystem. However, many challenges have surfaced during this pandemic through fragmented health systems and the lack of interoperability in many healthcare systems worldwide has led to an unprecedented strain on hospitals and an overwhelmed clinical workforce. To truly move this field forwards in the new normal, it requires novel and agile ways of working and collaboration, step-change improvements in digital transformation strategies, and better integration of data, technologies and processes, so that the digital health solutions that are designed and developed can address real-world problems. The primary goal of the International Journal of Digital Health is to serve as a cross-disciplinary, collaborative resource for all who are interested in this area, so that advancements in digital health can be expedited. We encourage all submissions in this area so that a robust evidence base can be built in digital health.

CORRESPONDING AUTHOR:

\section{Mr. Thomas Franchi, MSc, FHEA MAcadMEd}

The Medical School, The University of Sheffield, Beech Hill Road, Sheffield, S10 2RX, United Kingdom

tpffranchi1@sheffield.ac.uk

\section{KEYWORDS:}

Digital health; digital transformation; digital healthcare innovation

TO CITE THIS ARTICLE: Yap KYi-L, Liu J, Franchi T, Agha RA. The Launch of the International Journal of Digital Health: Ensuring Digital Transformation in Healthcare Beyond Covid-19. International Journal of Digital Health. 2021; 1(1): 2, 1-5. DOI: https://doi. org/10.29337/ijdh.27 
In recent years, digital health technologies have been evolving rapidly and there has been much interest and excitement surrounding their use in healthcare. The electronic medical record (EMR) maturity model by the Healthcare Information and Management Systems Society (HIMSS) and meaningful use initiatives of similar efforts by various governments have significantly driven healthcare organizations worldwide to adopt EMRs in the past 15 years. Adoption and optimization of EMRs was the focal point to information technology (IT) development for most of the healthcare organizations in the past decade. Built upon clinical documentation, digital imaging, online ordering, bar coding, device interface and many other technologies to enable paperless operations, cutting edge organizations have also used digital technology enabled process reengineering to develop and optimize closed loop clinical processes, and develop point-of-care clinical decision support and managerial decision support capabilities to achieve healthcare excellence.

As a result of the digitalization of transactions and documentations, big volumes of data have been captured and stored in various healthcare organizations. Traditional concerns over data confidentiality and patient privacy, along with insufficient interoperability and clearly limited connectivity in healthcare organizations have severely jeopardized the use of healthcare big data to develop artificial intelligence and achieve continuum of care. Patient experiences with healthcare organizations, by and large, have fallen behind from many other industries. It is suggested that healthcare is the last industry to be shaken up by digital technologies.

With the widespread use of cloud computing, big data, artificial intelligence, mobile technologies, wearable devices, internet of things, robotics, 3D-printing and $5 \mathrm{G}$ technologies, healthcare organizations have started embracing digital and communication technologies to extend their reach to patient populations outside their organizational boundaries in recent years. Patient engagements, clinical population management, telemedicine, telehealth and virtual care have started gaining momentum in the past five years. Excitement in big data and artificial intelligence has also engulfed the clinical community with the promise of a more personalized care experience through precision medicine, along with genetic advancement.

Yet, the progress to transform healthcare by adopting digital technologies has been slower than what many of us had anticipated. According to recent reports by HIMSS [1-2], prior to the coronavirus disease (Covid-19) outbreak, digital health technologies, such as telehealth, were used mainly for non-clinical purposes. Healthcare facilities generally used telehealth to conduct training and education (53\%), video-consultations (40\%), remote monitoring (43\%) and tele-triage (34\%). Although over $70 \%$ of telehealth services were used for chronic disease patients, telehealth remains underutilized in acute care.
Other statistics also suggested a decreasing adoption trend of digital health technologies by consumers at the end of 2019 [3], with one-third of US consumers were not using digital tools to manage their health. Furthermore, there was a decreased use of mobile health (mHealth) apps and wearable devices, particularly among the younger generations, possibly because the fitness tracker fad had begun to die down, but also due to more wearable devices (such as smartwatches) that were entering the market.

The Covid-19 pandemic has brought about a significant jump in the adoption of digital health innovations worldwide. Healthcare providers are now seeing a tremendous increase in the number of patients through telehealth [4]. Attitudes and behavior towards the adoption of digital health innovations are also changing. Over half of healthcare providers now view telehealth more favorably than they did pre-Covid-19 and consumers are more likely to use telehealth moving forward [4-6]. Among international efforts to contain the disease through contact tracing, social distancing and quarantines [7], it is clear that there is an accelerated need, and a global shift, to implement digital health solutions in the most efficient manner in order to provide optimal patient care and yet maintain patient safety.

Many organizations are rethinking their strategies to find better ways to adapt and improve the health ecosystem. However, many challenges have surfaced during this pandemic through fragmented health systems [1]. The lack of interoperability in many healthcare systems worldwide has led to an unprecedented strain on hospitals and an overwhelmed clinical workforce. Without relevant and reliable health-related data, healthcare professionals cannot manage patients optimally, and resource planning for information technology services are also challenged. Furthermore, with the international phenomenon of the 'Silver Tsunami', many elderly patients are falling through the cracks in various social healthcare systems in the community.

Covid-19 has also brought to light the digital divide in many countries - certain groups of patients, for example, the elderly and those from lower income households and in rural areas, are not benefitting from digital health services. Patients with chronic medical conditions who need to continue their care are forced to lower their expectations for virtual care services during the pandemic. In addition, the advent of new technology companies and virtual care services using chatbots and artificial intelligence has led to cybersecurity concerns regarding patients' health information [3]. A bad digital experience with a healthcare provider does not only demotivate patients from actively managing their health, but also leads to poorer health outcomes. These challenges have shown that there is a need to improve the digital health ecosystem and cultivate awareness and pro-digital health cultures in the workplace and among the public. 
Digital technologies and communication are playing very strong roles in flattening the world. Some of the developing economies have benefited tremendously, by adopting digital and communication technologies, to rapidly catch up with their developed counterparts in every segment of society. Growth markets have reported fewer barriers to adoption of digital and communication technologies, compared to their more mature market counterparts [8]. In time to come, we shall see the same happen to healthcare organizations. With a rapidly increasing number and variety of digital health innovations and technology players coming into the scene, now is the right time to document the digital health transformations happening globally through an evidence-based approach. Many of these innovations have not been studied formally in clinical settings or have only undergone pilot programs. Future digital health trends envisioned in the post-Covid-19 era include newer mHealth and tele-modalities, virtual and augmented reality, 3D-printing and additive manufacturing, artificial intelligence and robotics, medical drones, in-silico clinical trials, digital therapeutics and precision medicine, among others [9-10]. Governments are refocusing their national digital health strategies. Various organizations are seeing the need to develop new health technology implementation models and assessment frameworks $[1,11-12]$. With over $65 \%$ of employers globally who are more likely to invest in digital health innovations in the next five years [8], it is crucial that digital health innovations are effective, safe and affordable in the long term. As such, a multidisciplinary approach is required, involving stakeholders from a wide variety of domains, including medicine, nursing, pharmacy and the pharmaceutical sciences, allied health, computer science and informatics, data science and analytics, digital media, engineering, psychological and behavioral sciences, public health, and health economics and management. It is our belief that the willingness and ability to effectively adopt digital technologies, wellguided by evidence and best-practice experiences, will be one of the core competencies for modern healthcare organizations. The exchange and sharing of such knowledge in the global healthcare community will also be an incubator to many future successes.

To truly move this field forwards in the new normal, it requires novel and agile ways of working and collaboration, step-change improvements in digital transformation strategies, and better integration of data, technologies and processes, so that the digital health solutions that are designed and developed can address real-world problems. This is the primary goal of the International Journal of Digital Health (IJDH) - to serve as a cross-disciplinary, collaborative resource for all who are interested in this area, so that advancements in digital health can be expedited. As such, we encourage stakeholders to participate in the digital healthcare innovation cycle for all digital health solutions that are developed, so that these solutions can be applied, evaluated, implemented and supported in healthcare practices (Figure 1). The ultimate goal is to broaden access and improve convenience to care, enhance patient outcomes and safety, as well as move towards a more efficient healthcare system globally.

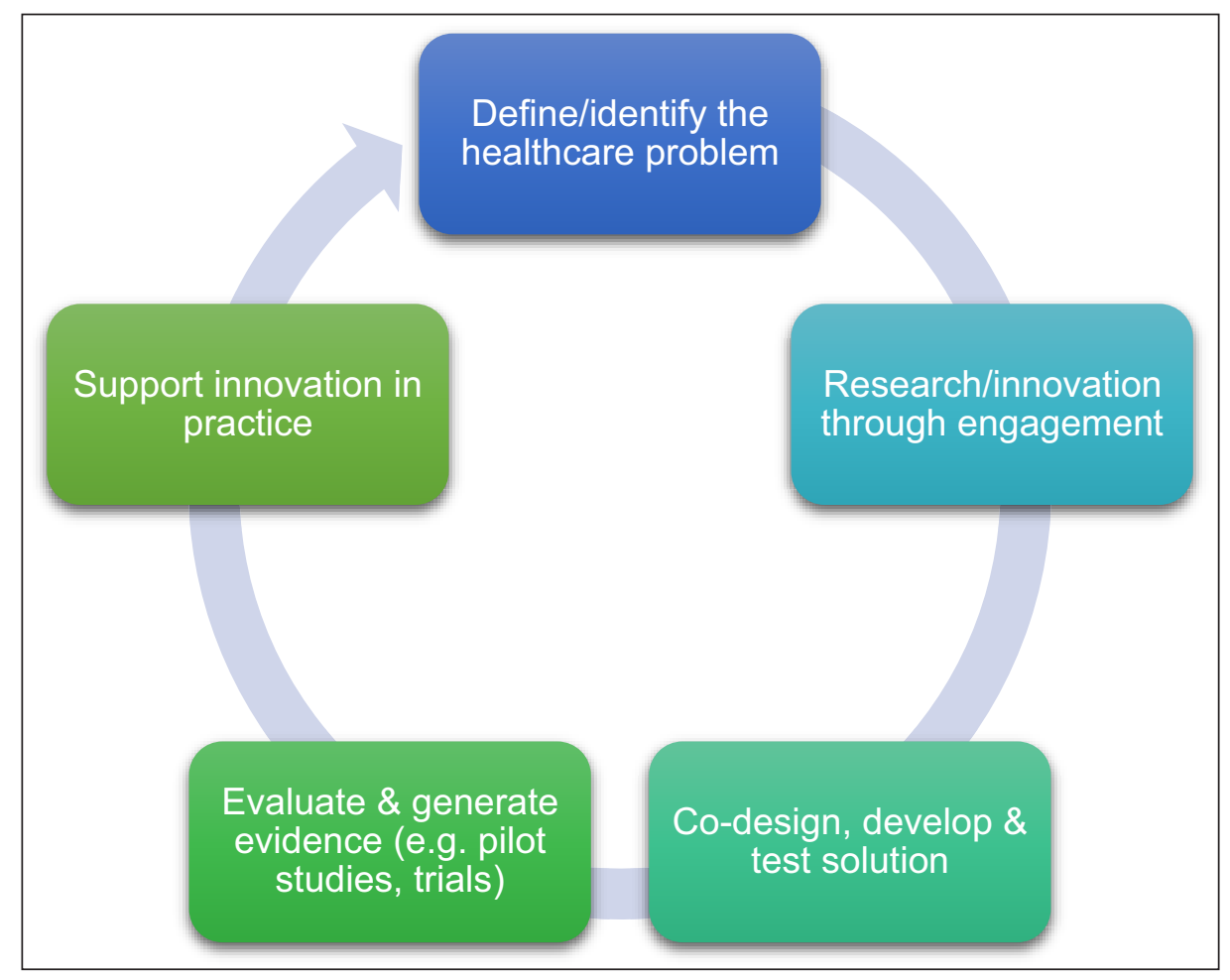

Figure 1 The digital healthcare innovation cycle. 
With the support of its deeply international and highly experienced Editorial Board, which spans academia, government and industry, IJDH strives to provide an international, peer-reviewed and open access platform to propel the digital transformation of healthcare and stimulate practice-changing research. We have the support of the IJS Publishing Group, which has nearly two decades of experience in successfully building and growing a portfolio of 10 journals. We therefore invite the global future-thinking community to contribute to its rigorous output, in the form of original research manuscripts, review articles, editorials, quantitative and qualitative studies, commentary and opinion pieces, digital health economic analyses, and other article types. The full aims and scope of the journal, alongside detailed instructions for authors, can be viewed at www. ijdigitalhealth.com. We eagerly await your submissions, support and suggestions.

\section{Dr Kevin Yi-Lwern Yap, Co-Editor-in-Chief} Dr Jilan Liu, Co-Editor-in-Chief

Mr Thomas Franchi, Assistant Managing Editor

Dr Riaz Ahmed Agha, Managing and Executive Editor

\section{COMPETING INTERESTS}

The authors are senior members of this journal's editorial board.

\section{AUTHOR AFFILIATIONS}

Kevin Yi-Lwern Yap (D) orcid.org/0000-0001-7322-4396 International Journal of Digital Health, United Kingdom; La Trobe University, Australia

\section{Jilan Liu}

International Journal of Digital Health, United Kingdom; HIMSS Greater China, United States

Thomas Franchi (D) orcid.org/0000-0003-2565-8098 International Journal of Digital Health, United Kingdom; The University of Sheffield, United Kingdom

Riaz Ahmed Agha (D) orcid.org/0000-0002-9348-0095 International Journal of Digital Health, United Kingdom; Harley Clinic, United Kingdom

\section{REFERENCES}

1. von Grätz PG, Ford P, Lovell T, Minion L, Rouger M, Barry N, et al. HIMSS Insights 8.4. COVID-19 and Beyond. Healthcare Information and Management Systems Society, Inc. 2020. https://indd.adobe.com/view/7c5b23ba-62aa4289-8774-5b064569ca8e (accessed 26 Jan 2021).

\section{Healthcare Information and Management Systems}

Society. E-health trendbarometer - Telehealth adoption in Europe. HIMSS Insights Special Edition. 2020; 1-67. https:// adobeindd.com/view/publications/4784c56b-465a-4ce5- 92ed-5f46bcdf6301/7cn6/publication-web-resources/ pdf/200611_Insights_SpecialEdition_AT.pdf, (accessed 26 Jan 2021).

3. Safavi K, Kalis B. How can leaders make recent digital health gains last? Re-examining the Accenture 2020 Digital Health Consumer Survey: US findings, Accenture; 2020. https://www.accenture.com/_acnmedia/PDF-130/ Accenture-2020-Digital-Health-Consumer-Survey-US.pdf (accessed 26 Jan 2021).

4. Bestsennyy 0, Gilbert G, Harris A, Rost J. Telehealth: A quarter-trillion-dollar post-COVID-19 reality? https://www.mckinsey.com/industries/ healthcare-systems-and-services/our-insights/telehealtha-quarter-trillion-dollar-post-covid-19-reality\#, 2020 (accessed 26 Jan 2021).

5. Bath K, Johnson J. Taskforce's Practice Management Cluster Group, COVID-19 Telehealth survey results, ASHM's Taskforce on BBVs, Sexual Health and COVID-19 Document. 2020; 1-5. https://ashm.org.au/covid-19/clinical-care/covid19-telehealth-survey-results-clinical-care/, (accessed 26 Jan 2021).

6. Lee K, Zafra M, Bay J. COVID-19 makes Singapore's digital health "on demand", 2020. https://www.mmc. com/insights/publications/2020/august/covid-19-makessingapore-s-digital-helath--on-demand-.html (accessed 26 Jan 2021).

7. John Leon Singh H, Couch D, Yap K. Mobile Health Apps That Help With COVID-19 Management: Scoping Review. JMIR Nurs. 2020; 3: e20596. DOI: https://doi. org/10.2196/20596

8. Friedman L, Laverock A, Umland B, Glick S, Lehman v, Passmore N. Health On Demand Global Report, Marsh \& McLennan Companies, 2020. https://www.oliverwyman. com/content/dam/oliver-wyman/v2/publications/2020/jul/ Health\%20on\%20Demand\%20-\%20Global\%20Report\%20 2020.pdf (accessed 26 Jan 2021).

9. Meskó B. The top 5 practical digital health technologies in the fight against COVID-19: An infographic https:// medicalfuturist.com/the-top-5-practical-digital-healthtechnologies-in-the-fight-against-covid-19-an-infographic/, 2020 (accessed 26 Jan 2021).

10. Meskó B, Dhunnoo P. The Medical Futurist's Guide to Invest in Digital Health, Leanpub, 2020. https://leanpub.com/ digital-health-investments (accessed 26 Jan 2021).

11. Greenhalgh T, Maylor H, Shaw S, Wherton J, Papoutsi C, Betton V, et al. The NASSS-CAT tools for understanding, guiding, monitoring, and researching technology implementation projects in health and social care: Protocol for an evaluation study in real-world settings. JMIR Res Protoc. 2020; 9: e16861. DOI: https://doi. org/10.2196/16861

12. Haverinen J, Keränen N, Falkenbach P, Maijala A, Kolehmainen T, Reponen J. Digi-HTA: Health technology assessment framework for digital healthcare services. Finnish Journal of eHealth and eWelfare. 2019; 11: 326-341. DOI: https://doi.org/10.23996/fjhw.82538 
TO CITE THIS ARTICLE:

Yap KYi-L, Liu J, Franchi T, Agha RA. The Launch of the International Journal of Digital Health: Ensuring Digital Transformation in Healthcare Beyond Covid-19. International Journal of Digital Health. 2021; 1(1): 2, 1-5. DOI: https://doi.org/10.29337/ijdh.27

Submitted: 10 February 2021 Accepted: 14 February 2021 Published: 04 March 2021

COPYRIGHT:

(c) 2021 The Author(s). This is an open-access article distributed under the terms of the Creative Commons Attribution 4.0 International License (CC-BY 4.0), which permits unrestricted use, distribution, and reproduction in any medium, provided the original author and source are credited. See http://creativecommons.org/licenses/by/4.0/.

International Journal of Digital Health is a peer-reviewed open access journal published by IJS Publishing Group.

IJS Press 